\title{
SIMULATING MULTIPLE QUANTUM WELL SOLAR CELLS
}

\author{
James P. Connolly ${ }^{a}$ Jenny Nelson ${ }^{a}$ Keith W.J. Barnham ${ }^{a}$ lan Ballard ${ }^{a}$ C.Roberts ${ }^{a}$ J.S. Roberts ${ }^{\mathrm{c}}$ C.T.Foxon ${ }^{\mathrm{d}}$ \\ a Blackett Laboratory, Imperial College of Science, Technology and Medicine, London SW7 2BZ \\ b Centre for Electronic Materials and Devices, Imperial College, London SW7 2BZ UK \\ c EPSRC III-V Facility, University of Sheffield, Sheffield S1 3JD UK \\ d University of Nottingham, Nottingham N67 2RD UK
}

Key words: Quantum well solar cell, dark current, light current, efficiency

\section{1 abstract}

The quantum well solar cell (QWSC) has been proposed as a route to higher efficiency than that attainable by homojunction devices. Previous studies have established that carriers escape the quantum wells with high efficiency in forward bias and contribute to the photocurrent. Progress in resolving the efficiency limits of these cells has been dogged by the lack of a theoretical model reproducing both the enhanced carrier generation and enhanced recombination due to the quantum wells. Here we present a model which calculates the incremental generation and recombination due to the QWs and is verified by modelling the experimental light and dark current-voltage characteristics of a range of III-V quantum well structures. We find that predicted dark currents are significantly greater than experiment if we use lifetimes derived from homostructure devices. Successful simulation of light and dark currents can be obtained only by introducing a parameter which represents a reduction in the quasi-Fermi level separation.

\section{Introduction}

The quantum well solar cell (QWSC) [1] consists of a $p-i-$ $n$ structure with nm-wide regions of low bandgap (quantum wells) in the nominally undoped intrinsic region where carriers occupy discrete energy levels.

Experiment has shown ([2], [3]) that photo-excited electrons and holes in these quantum wells escape with high efficiency in forward bias and contribute to the photocurrent. If the photocurrent enhancement exceeds the dark current increase this may be a highly efficient solar cell design.

Detailed balance arguments in the radiative limit [4] have suggested the $\mathrm{V}_{\mathrm{oc}}$ is determined by the lowest absorption energy which is the well bandgap. Further work [5] however indicates that efficiency enhancements are possible over single bandgap designs in non-radiative dominated structures. This has been confirmed by work ([6] [7]) which showed smaller quasi-Fermi level (QFL) separation in the wells than in the barriers. This suggests that significant efficiency enhancements are possible with this solar cell design.

This paper describes QWSC photocurrent and dark current calculations with a view to establishing whether the effect of enhanced photocurrent overcompensates the dark current losses and secondly modelling the overall device performance. The photocurrent is calculated from the diffusion equation applied to photogenerated minority carriers in bulk and well material and is well understood. The dark current aspect is a

1 Electronic mail: j.connolly@ic.ac.uk
Shockley-Read-Hall (SRH) calculation including an ideal diode component. The model examines the extent to which the dark current behaviour of QWSCs can be characterised in terms of the quantum well density of states without considering escape and capture rates from the quantum wells. Furthermore, it explores the extent to which the QWSC dark current is determined by the SRH recombination rates at the centre of the intrinsic region, and for many wells where this methodology is expected to be accurate. This is explored by modelling a range of experimetally measured dark currents in the well characterised $\mathrm{GaAs} / \mathrm{Al}_{\mathrm{x}} \mathrm{Ga}_{1-\mathrm{x}}$ As system.

\section{QWSC current voltage model}

The program "SOL" calculates the incremental light and dark currents due to the quantum wells. It will be shown that the changes in photocurrent and dark current can both be reduced to functions of the quantum well densities of states.

The photocurrent model of the QWSC consists of a spectral response calculation as a function of bias, cell dimensions and quantum well energy spectrum. Cell photocurrents can then be calculated for different incident light spectra and power levels. This has been described for the GaAs $/ \mathrm{Al}_{\mathrm{x}} \mathrm{Ga}_{1-\mathrm{x}} \mathrm{As}$ $\mathrm{GaAs} / \mathrm{In}_{\mathrm{x}} \mathrm{Ga}_{1-\mathrm{x}} \mathrm{As} \mathrm{InGaP}_{\mathrm{x}} \mathrm{Ga}_{\mathrm{x}} \mathrm{AsP}$ and $\mathrm{InGa}_{0.53 \mathrm{x}} \mathrm{As}_{\mathrm{x}} \mathrm{P}$ materials systems [2], [3].

The dark currents measured in QWSC structures in these materials show idealities close to 2. This is characteristic of SRH recombination in the depletion layer. The dark current model applied here is a simplified implementation of a self consistent model by Nelson et al [8] which has succesfully described the dark currents of single quantum well cells for a range of single well positions and $i$ region background doping levels.

The carrier generation rate $G$ at wavelength $\lambda$, position $x$, incident photon flux $F, R$ the surface reflectivity and absorption coefficient $\alpha$ is defined as

$$
G(x, \lambda)=F(\lambda)(1-R(\lambda)) \alpha(x) e^{-\int_{0}^{x} \alpha x d x}
$$

In the quantum wells the generation is calculated from Fermi's golden rule governing transition probabilities between electron and hole well states. These are found by solving the effective mass equation for electrons and holes confined in the QW within the envelope function approximation. Omitting constants for brevity it is written as

$$
\alpha_{q w} \sim \sum_{j n=1, j p=1}^{N \text { states }} M^{2} H\left(E_{j n}-E_{j p}-\hbar \omega\right)
$$

where the double sum is over initial and final valence and conduction band states labelled $j n$ and $j p, M$ is the transition Hamiltonian, $H$ is the Heaviside step function delta, $E_{j n}$ and 
$E_{j p}$ are the energy levels of the initial and final quantum well states.

The photocurrent $J_{i}$ due to the QW at any $\lambda$ is found by integrating $G$ across the QW layers and assuming $100 \%$ escape. The QW spectral response is added on to that of the bulk regions of the device calculated as in [2].

Figure 1 shows the calculated and measured $\mathrm{QE}$ for an $\mathrm{Al}_{\mathrm{X}}$ $\mathrm{Ga}_{1-\mathrm{x}}$ As QWSC comprising 30 GaAs wells together with the same for a control sample which is identical but without wells. Under an standard AM1.5 spectrum the theoretical short circuit current $\left(J_{s c}\right)$ for this sample is $108 \mathrm{Am}^{-2}$ versus $116 \mathrm{Am}^{-2}$ calculated from the experimental SR. These results agree with calibrated results from NREL to within $3 \%$ in the worst case.

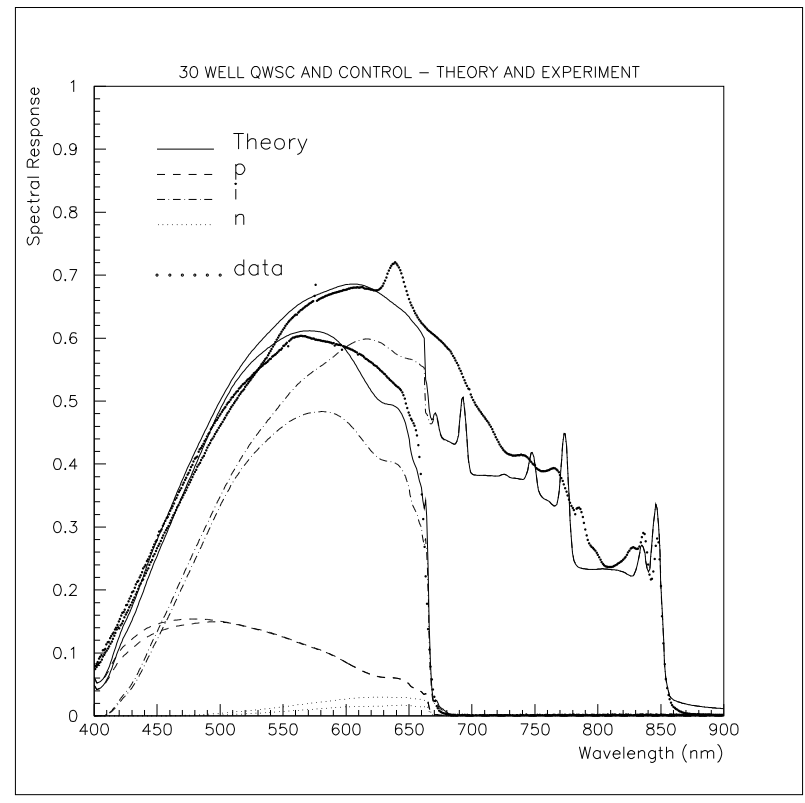

Fig. 1. Experimental and calculated $\mathrm{SR}$ of a 30 well $\mathrm{Al}_{\mathrm{x}} \mathrm{Ga}_{1-\mathrm{x}} \mathrm{As}$ sample with aluminium $x=30 \%$

The SRH [9] recombination rate $U(x)$ in the i region is given by

$$
U(x)=\frac{p n-n_{i}^{2}}{\tau_{n}\left(p+p_{t}\right)+\tau_{p}\left(n+n_{t}\right)}
$$

where where $p_{t}$ and $n_{t}$ are hole and electron populations in trap levels in the bandgap, $p$ and $n$ are hole and electron populations in the valence and conduction bands and $\tau_{p}$ and $\tau_{n}$ are their respective lifetimes.

Electron and hole populations in barrier regions of the intrinsic region are expressed in terms of the intrinsic population $n_{i}$, Fermi levels $E_{f}$ and the intrinsic energy $E_{i}$

$$
\begin{aligned}
& n_{b}=n_{i} e^{\left(\frac{E_{f n}-E_{i}}{K T}\right)} \\
& p_{b}=n_{i} e^{\left(\frac{E_{i}-E_{f p}}{K T}\right)}
\end{aligned}
$$

where $n_{i}$ is given by the effective masses of electrons and holes via the conduction and valence band effective densities of states and the law of mass action in equilibrium, and for $n_{b}$ the subscript $b$ refers to barrier material.

It has been shown [8] that the variation in electron and hole populations in well and barrier regions can be reduced to a difference in effective mass within the parabolic band approximation and a constant factor modifying the conduction and valence band densities of states which is determined by a sum over quantum well allowed states. These are written in terms of two functions $\theta_{p}$ and $\theta_{n}$. Electron and hole populations $n_{q}$ and $p_{q}$ in the wells are then given as follows in terms of the barrier concentrations

$$
\begin{aligned}
& n_{q}=n_{b} e^{\theta_{n}} \\
& p_{q}=p_{b} e^{\theta_{p}}
\end{aligned}
$$

The $\theta$ functions are as follows

$$
\begin{aligned}
& \theta_{n}=k T \ln \left[\frac{1}{L}\left(\frac{2}{N_{C}}\right)^{1 / 3} \frac{m_{n q}}{m_{n}} \sum_{j n=1}^{N} e^{\left(\frac{E_{c q}-E_{j n}}{K T}\right)}\right] \\
& \theta_{p}=k T \ln \left[\frac{1}{L}\left(\frac{2}{N_{V}}\right)^{1 / 3} \frac{m_{p q}}{m_{p}} \sum_{j p=1}^{N} e^{\left(\frac{E_{j p}-E_{j q}}{K T}\right)}\right]
\end{aligned}
$$

where $L$ is the quantum well width. $m_{n, p}$ are electron and hole masses in the barrier and $m_{n q, p q}$ are the masses in the well. These functions contain all information necessary about the quantum wells with the sole exception of carrier lifetimes. We note that they are symmetrical to the well photocurrent enhancement term of equation 2. Both involve a sum over well states and the necessary information is the same as in equation 2 and reduces to band structure data about the well relative to the barriers. The functions will not be symmetrical for electrons and holes because of different valence/conduction offsets and effective masses.

The contributions from neutral layers are included in terms of the ideal diode formalism The electron and hole Fermi-levels are set equal to the valence and conduction band edges. The depletion approximation is made with the result that photogenerated carriers are omitted in the dark current calculation. The dark current of the device in this picture is given by the integral of the SRH recombination rate over the depletion region including $\mathrm{p}$ and $\mathrm{n}$ layer depletion layers if present, incrememented by the ideal diode contribution from the charge neutral regions. An example SRH profile is shown in figure 2. The quantum wells show are step-like peaks in the profile, and clearly dominate the dark current of a QWSC.

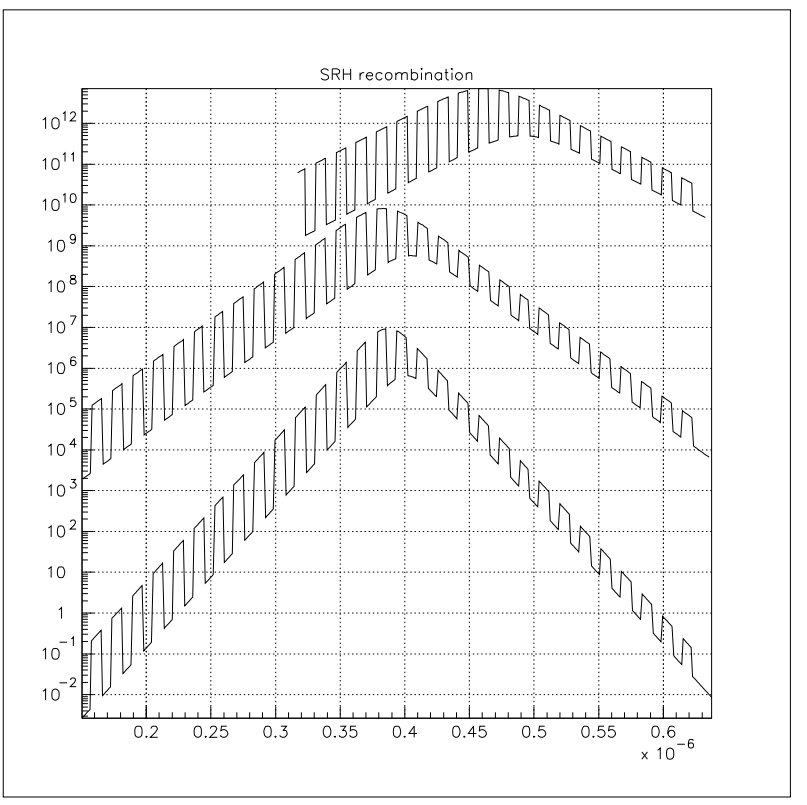

Fig. 2. SRH recombination profile for the 30 well QWSC of figure 1 for a biasses $0.8 \mathrm{~V}, 1.15$ and $1.5 \mathrm{~V}$, showing different $\theta_{n}$ and $\theta_{p}$ and the reduction of SCR width at high bias. 


\section{Experiment and Modelling}

Samples were grown by MBE and MOVPE in the Centre for Electronic Materials and Devices in Imperial College, Phillips Laboratories (Redhill) and the Sheffield III-V Semiconductor Facility. They take the form of $p-i-n$ diodes. Structures having GaAs wells in the $i$ region will be referred to as QWSCs and identical $p-i-n$ structures of homogeneous composition grown in the same growth run are termed controls. The experimental configuration has been described previously [2]. The fitting parameters for dark currents are the non radiative lifetimes. Estimates for these are drawn from studies of barrier and well controls which are $\mathrm{GaAs} / \mathrm{Al}_{\mathrm{x}} \mathrm{Ga}_{1-\mathrm{x}} \mathrm{As}$ and $\mathrm{GaAs}$ controls. These lifetimes are then applied to QWSC structures and the resulting prediction compared with experiment. Figure 3 shows modelled and calculated IV for $\mathrm{GaAs}$ and $\mathrm{Al}_{\mathrm{X}}-$ $\mathrm{Ga}_{1-\mathrm{x}}$ As controls. The upper curves shows a GaAs control. The model shows SRH and ideal dark currents and their sum. The onset of radiatively dominated behaviour is visible below the built in voltage of $1.44 \mathrm{~V}$ and is well described. The parameters for the ideal Shockley fit are the same as those used for the SR leaving the non radiative lifetimes as the only parameters. They are chosen equal in this case. Making them different shifts the SRH peak with position and changes the ideality of the dark current in disagreement with experiment. The lifetime of $10 \mathrm{~ns}$ is in close agreement with lifetimes used in previous work [8].

The lower curves shows similar data and theory for an $\mathrm{Al}_{\mathrm{x}}-$ $\mathrm{Ga}_{1-x}$ As control with a much shorter SRH lifetime of $0.6 \mathrm{~ns}$. Again this is in agreement with previous work by the same factor in a different sample. Modelling an identical control with aluminium mole fractions $x=20 \%$ yields a lifetime of $0.05 \mathrm{~ns}$.

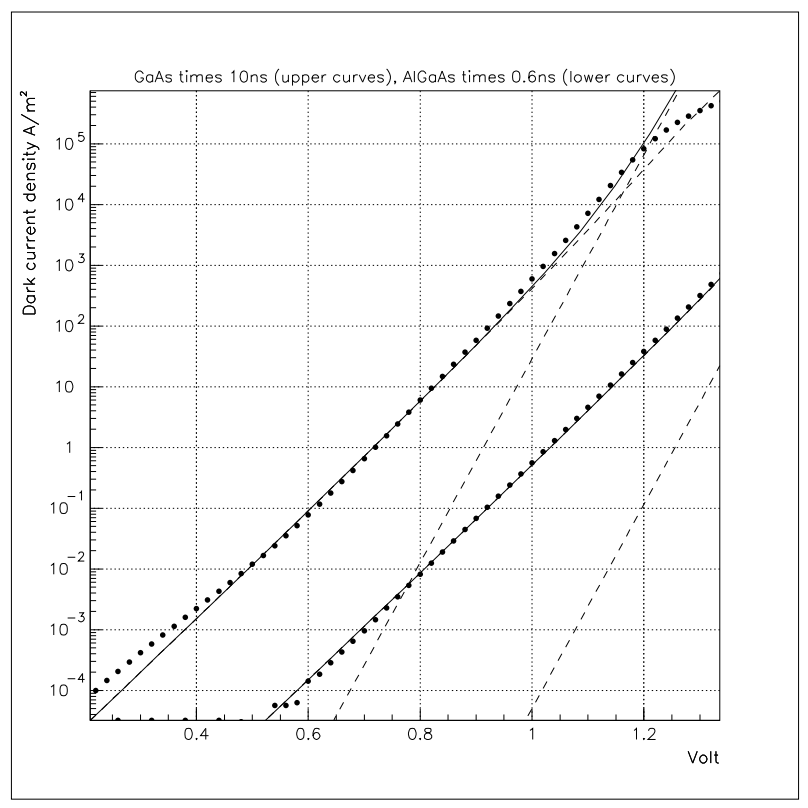

Fig. 3. GaAs control dark current and lifetimes

Figures 4 and 5 show dark current modelling for 30 well MBE grown QWSCs with Al composition of $20 \%$ and $33 \%$ and with lifetimes derived from relevant controls assuming similar material quality.

Figure 6 shows the same data for a 50 well QWSC with Al fractions $33 \%$. Fitting the data can again be achieved by reducing the QFL separation by the same shift.

We have observed that experiment systematically records a lower dark current than predicted by the control lifetimes. This

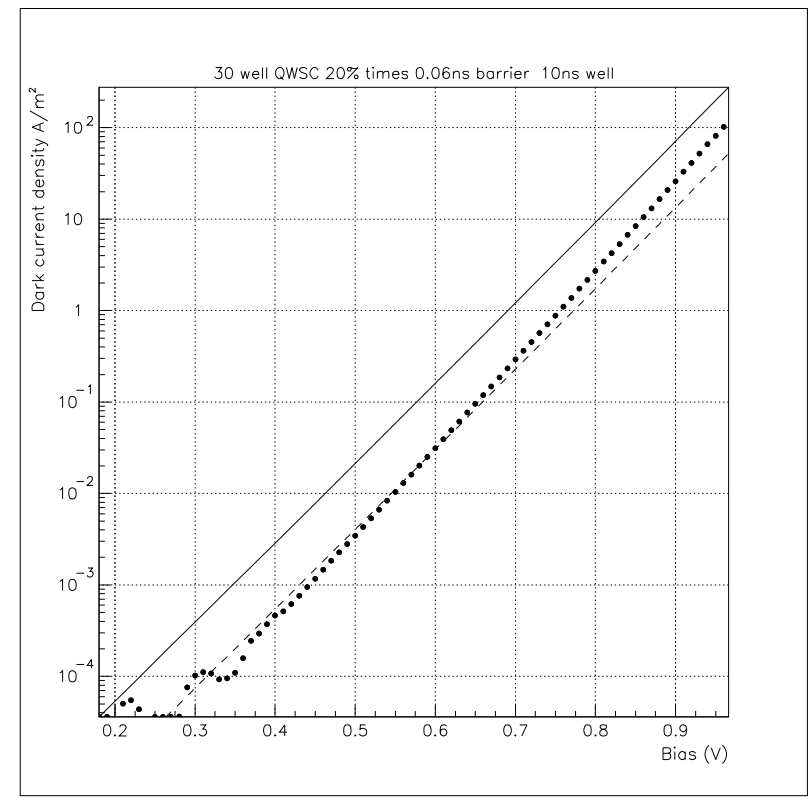

Fig. 4. 30 well QWSC AI $20 \%$ (dashed line modified $\delta E_{f}$ see text)

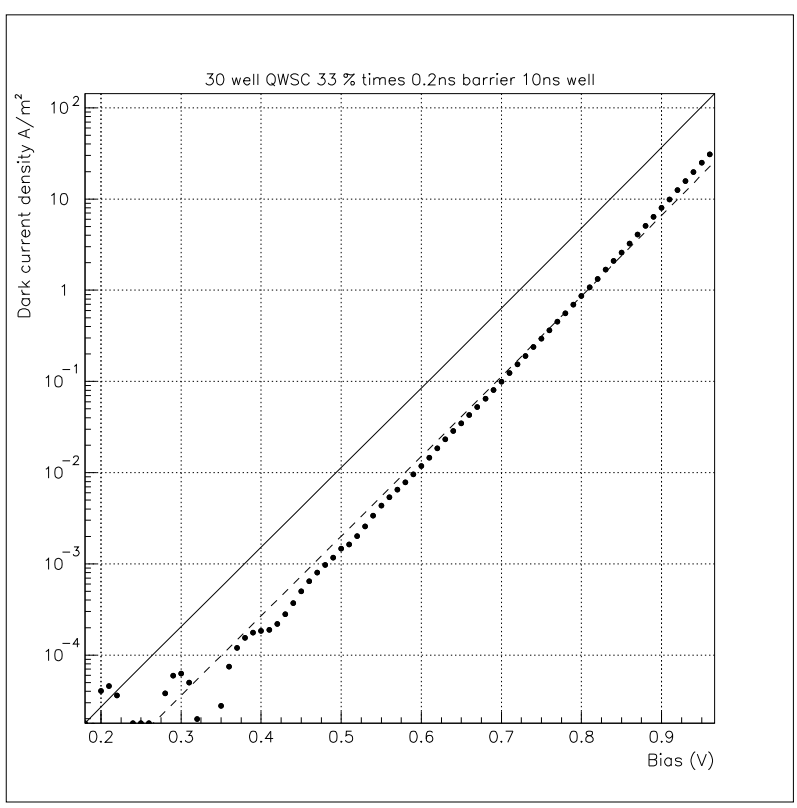

Fig. 5. 30 well QWSC AI 33\% (dashed line modified $\delta E_{f}$ see text)

can be explained in a variety of ways by the model. Longer lifetimes in the wells linearly decrease the dark current until the well lifetime is much longer than in the barrier. However there is no reason why the lifetimes in the QW should be greater than in bulk material.

The approach we have investigated is the possibility of a lower QFL separation $\delta E_{f}$ in the quantum wells. We find that a narrowing of the QWFL separation in the wells by a factor symmetrical for holes and electrons models all samples well, as shown by the dashed lines in graphs 4 to 6 despite the fact that the barrier bandgap varies significantly, and that one sample has 50 wells.

This reaffirms work [10] on the QFL separation which was found to be significantly smaller in SQW samples at $10 \mathrm{meV}$ 


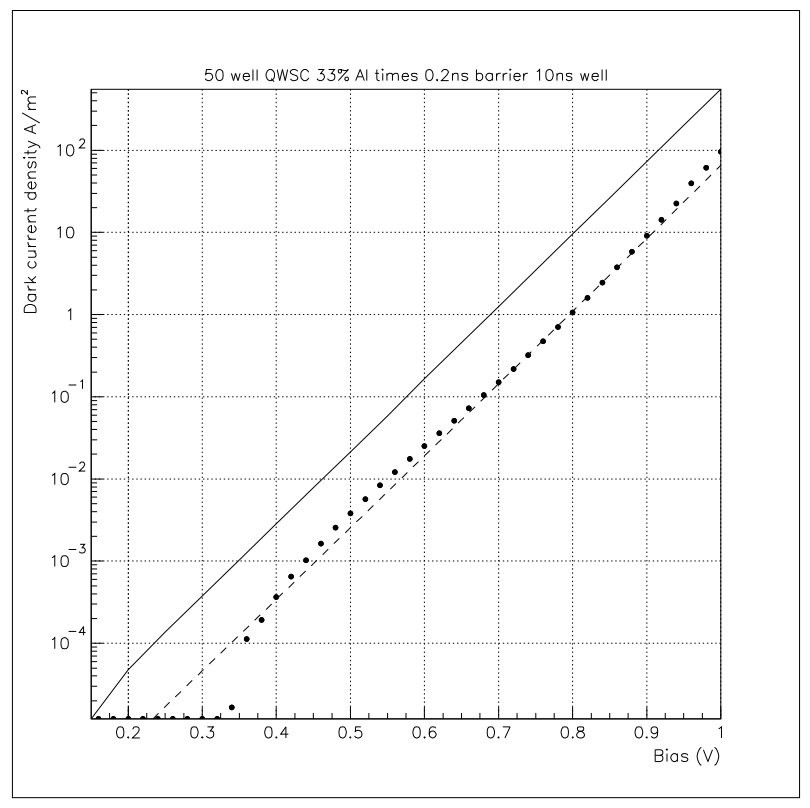

Fig. 6. 50 well QWSC Al 33\% (dashed line modified $\delta E_{f}$ see text)

\section{Conclusions}

The QWSC benefits from an increase in photogeneration in the wells which is counterpointed by recombination in the lower bandgap well regions. In order to study which effect is greater we study photocurrent and dark current and express the modifications to dark and photocurrent in terms of the quantum well density of states. The photocurrent from the wells is determined with no free parameters to good accuracy. The dark current for the control homojunctions is well understood. Modelling these gives us an estimate of carrier lifetimes in the SCR, together with verifying the transport parameters used in the $Q E$ calculation at the onset of ideal diode behaviour, if present.

The QWSC dark current depends on four lifetimes. We reduce this to two by assuming that hole and electron non radiative lifetimes are equal. In the absence of direct measurements we derive the barrier lifetimes from controls with the barrier composition. Similarly, controls with the well composition set an upper limit on the well lifetimes employed.

We see a systematic overestimation of the dark current, indicating that the well lifetimes in a QWSC system are longer than expected by one order of magnitude to a first approximation. This can be explained in terms of longer well lifetimes or a smaller QFL separation in the wells. This strongly suggests that these structures have the potential to be efficient photoconverters.

Finally we note that this formulation of the MQW dark current problem shows shortcomings because of the approximations made. This is particularly noticeable in the case of single well samples. A full treatment needs a self consistent approach to the Poisson equation.

This would be noticeable for single quantum well samples where the position of the quantum wells and the background doping is important. We note however that the treatment applies reliably to MQW systems studied here since dark current is determined mainly by the balance between QW and barrier material, in that on average the position of the wells is not critical. This is borne out by the range of $\mathrm{GaAs} / \mathrm{Al}_{\mathrm{x}} \mathrm{Ga}_{1-\mathrm{x}} \mathrm{As}$ samples examined.

\section{REFERENCES}

[1] Barnham KWJ and Duggan G, A New Approach to High Efficiency High-Bandgap Solar Cells, J. Appl. Phys, 67 (7), 1990

[2] M.Paxman, J.Nelson, K.W.J.Barnham, B.Braun, J.P.Connolly, C.Button, J.S.Roberts and C.T.Foxon Modelling the Spectral Response of the Quantum Well Solar Cell J.Appl.Phys. 74, 614 (1993).

[3] J.Barnes, J.Nelson, K.W.J.Barnham, J.S.Roberts, M.A.Pate, S.S. Dosanjh, R.Grey, M.Masseur and F.Ghiraldo, Characterisation of GaAs/InGaAs quantum wells using photocurrent spectroscopy, J. Appl. Phys. 79 7775-9 (1996)

[4] G. L. Araujo et al., Proc. 12th European Photovoltaic Solar Energy Conference, pp. 14811484 (1994)

[5] Keith Barnham, James Connolly, Paul Griffin, Guido Haarpaintner, Jenny Nelson, Alexander Zachariou, Jane Osborne voltage enhancement in Quantum Well Solar Cells, J. apply. Phys 801201 (1996)

[6] Jenny Nelson, Benjamin Kluftinger, Ernest S.M.Tsui, Keith Barnham et al., Quasi-Fermi Level Separation in Quantum Well Solar Cells, 13th European Photovoltaic Energy Conference, Nice, pp150 (1996)

[7] E.S.M Tsui, J.Nelson, K.W.J. Barnham, C.Button and J.S.Roberts, Determination of the Quasi Fermi level separation in single quantum well $p-i-n$ diodes J. Appl. Phys. 80 (7) (1996)

[8] Jenny Nelson, lan Ballard, Keith Barnham, James P. Connolly, Effect of quantum well location on single quantum well $p-i-n$ photodiode dark currents, J. Appl. Phys, 86 (10), 1999

[9] Shockley, W. T. Read, and r. N. Hall, Phys. Rev. Lett. 87, 835 (1952)

[10] Jenny Nelson, Jenny Barnes, Nicholas Ekins-Daukes, Benjamin Kluftinger, Ernest Tsui and Keith barnham Observation of suppressed radiative recombination in single quantum well $p-i-n$ photodiodes J.Appl.Phys. 82, 6240 (1997). 
$30 \mathrm{MQW}$ at $36 \%$ Al barrier $0.6 \mathrm{~ns}$ well $10 \mathrm{~ns}$

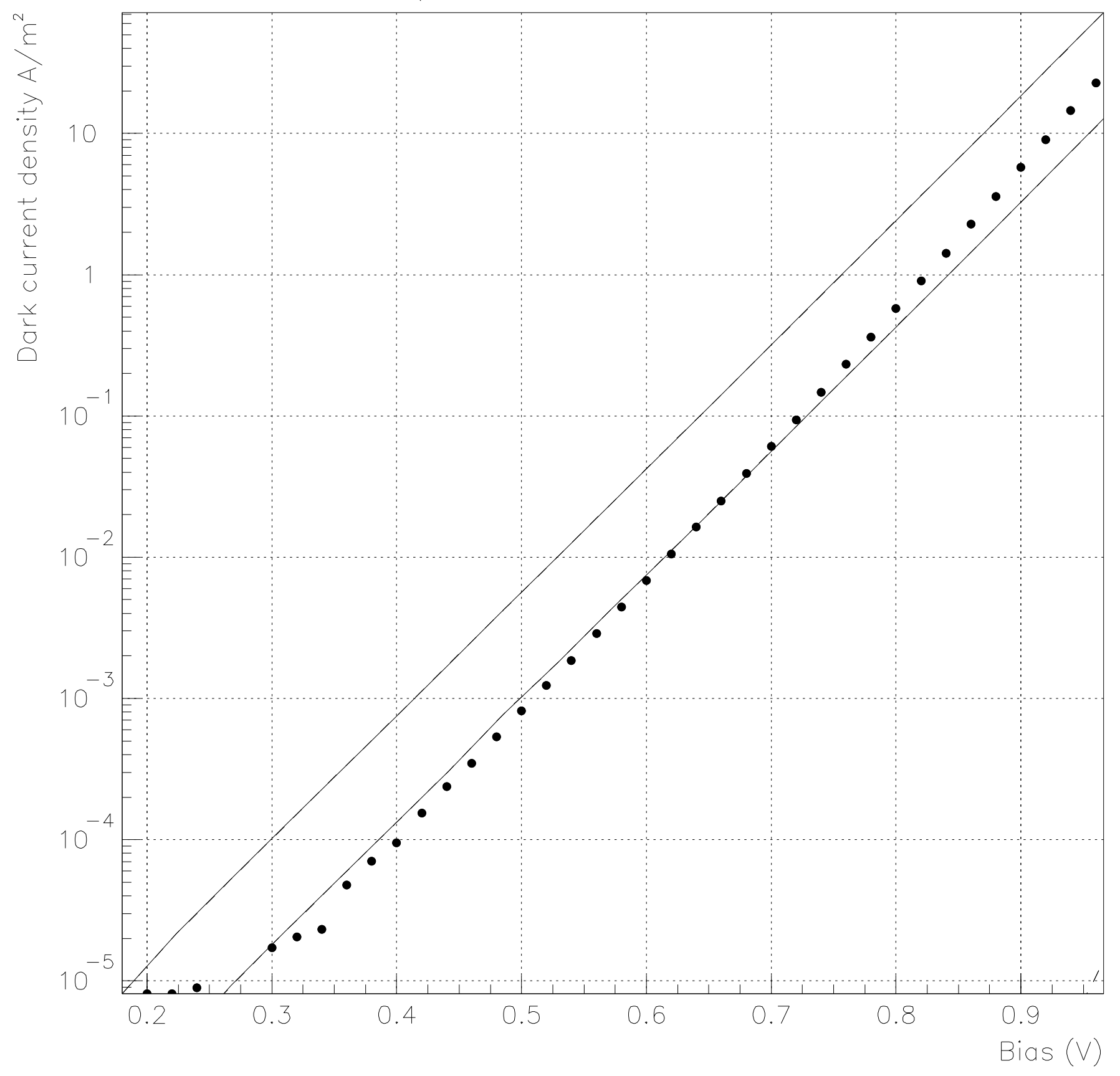




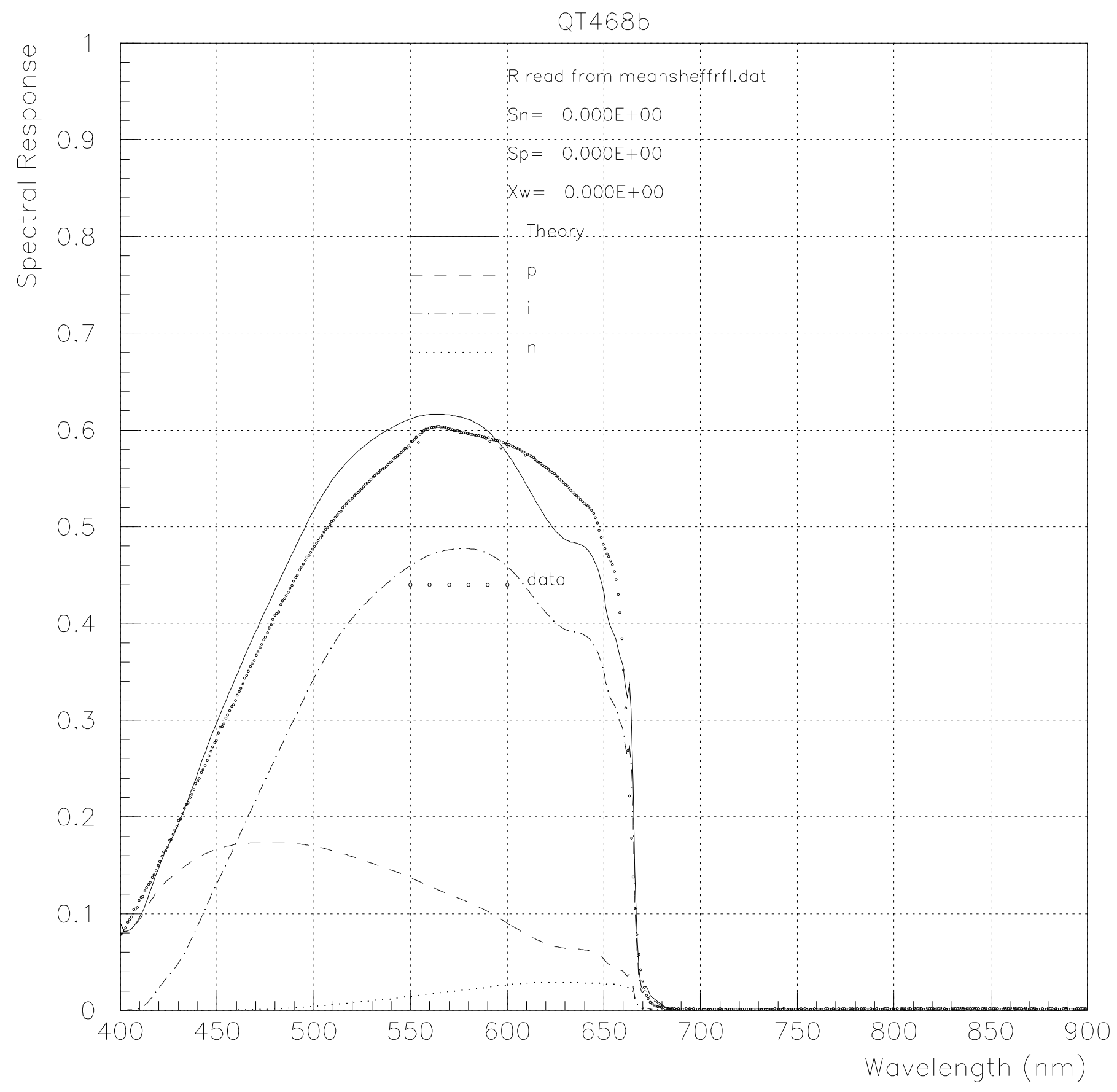


Hole and electron populations

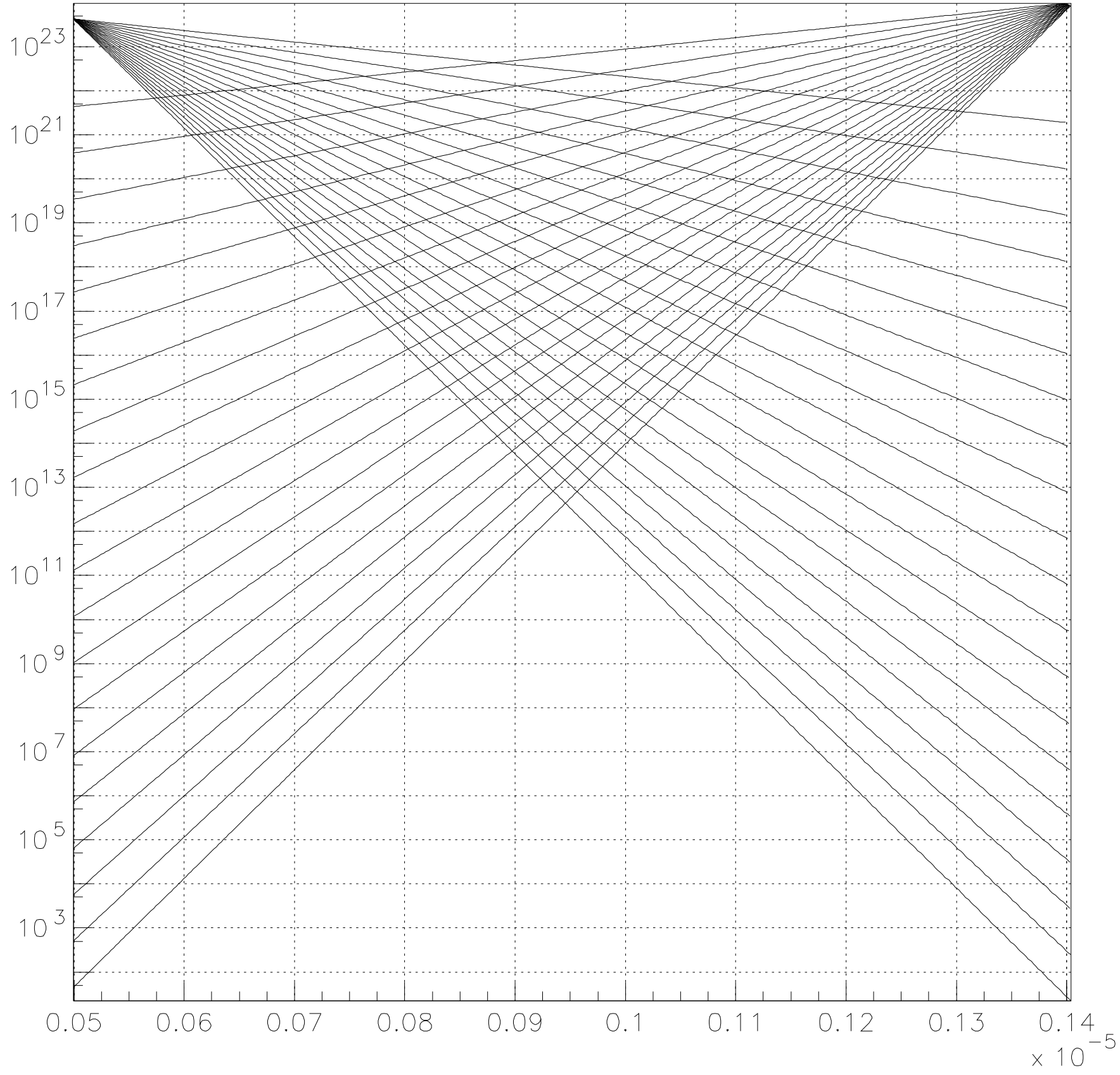




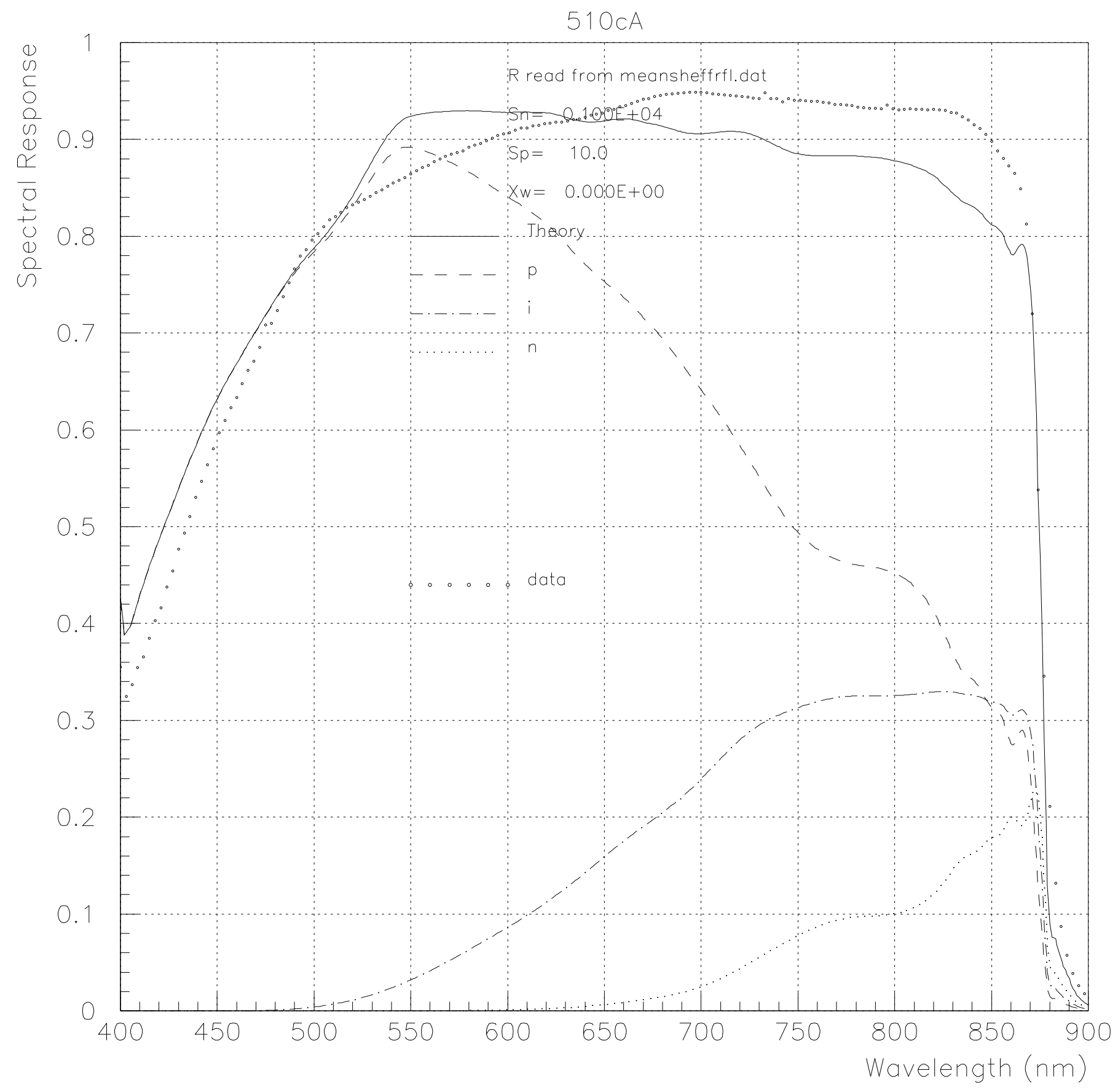


SRH recombination

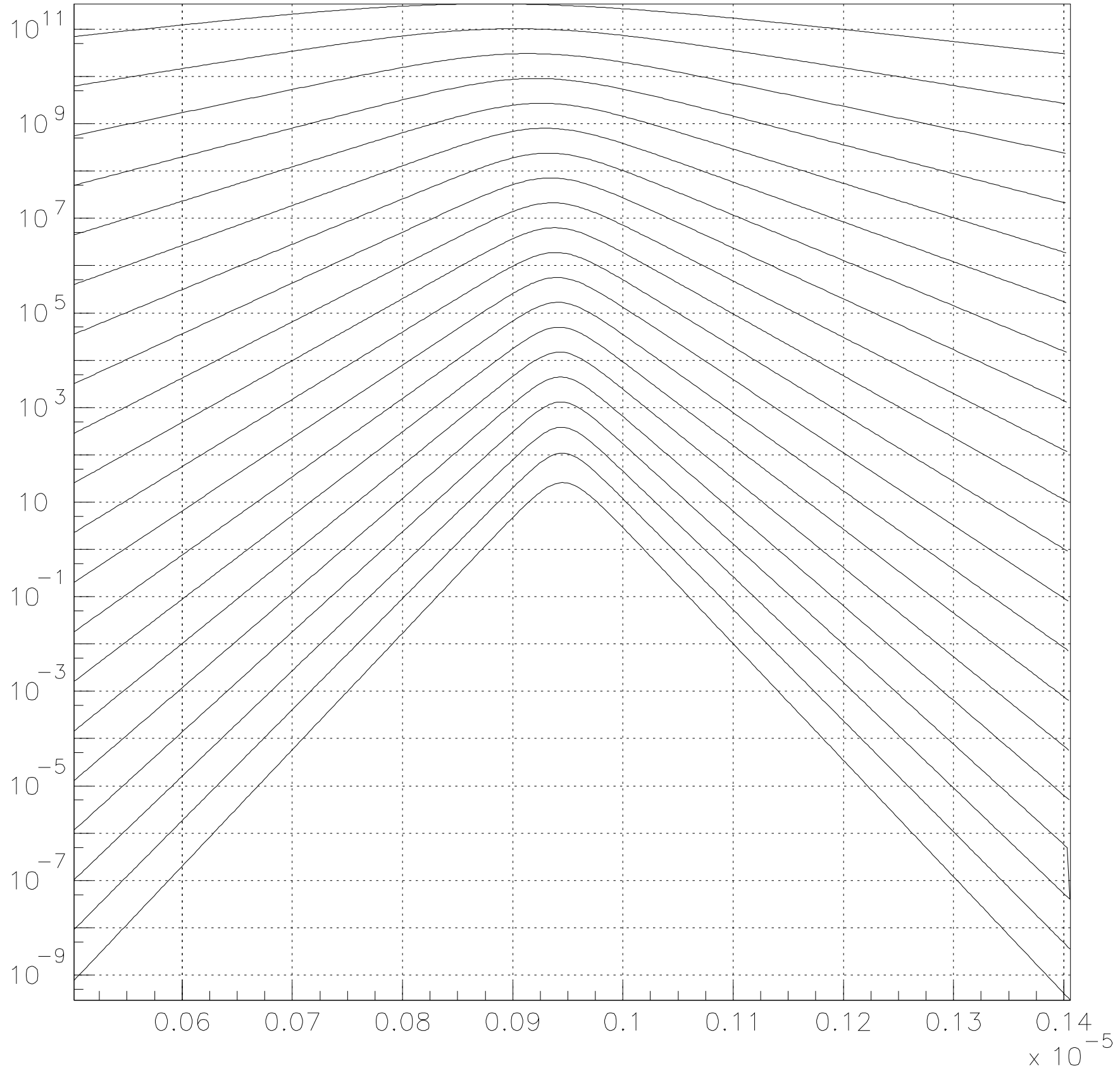




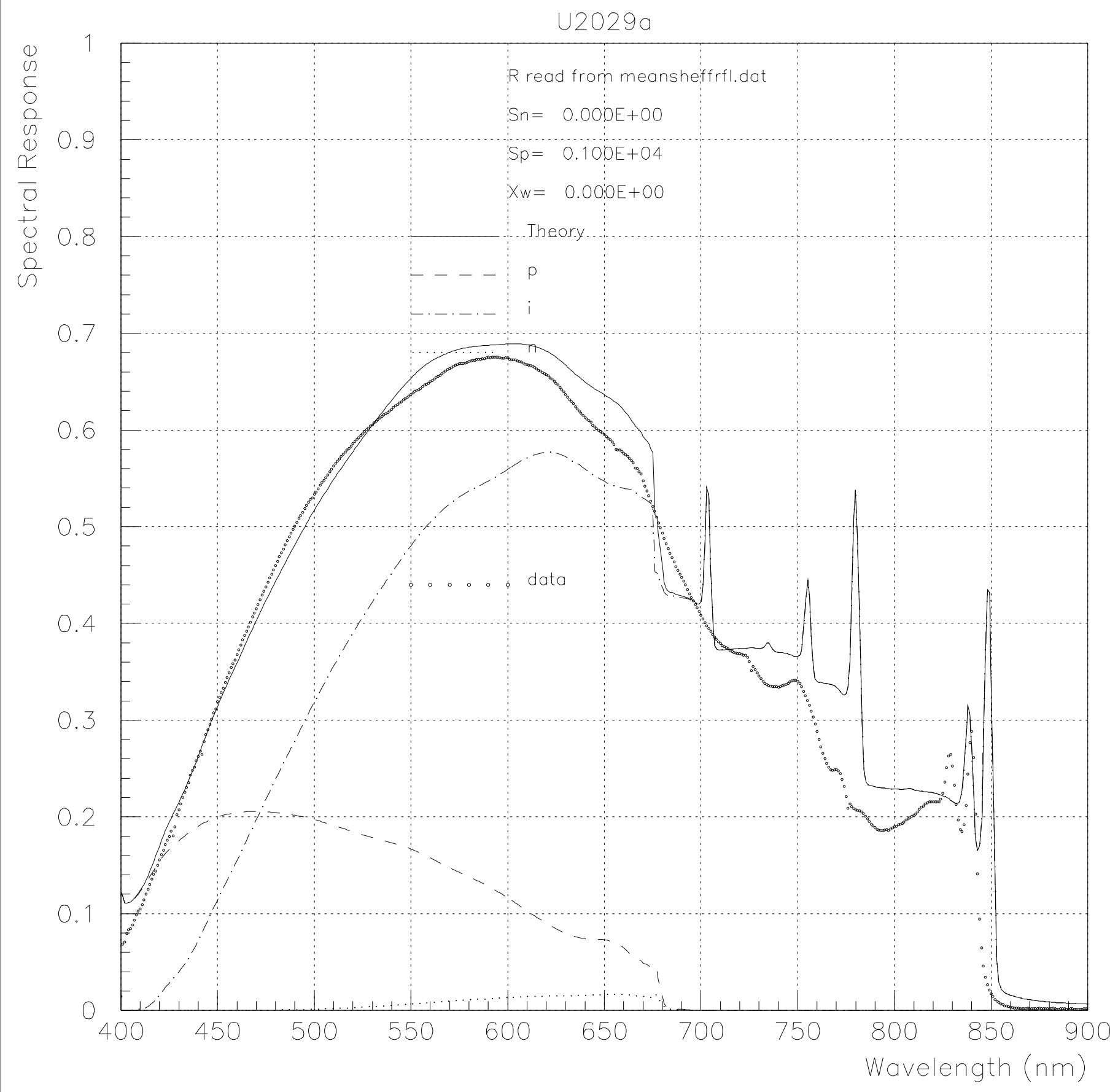


Times ( $p b, n b: \quad 0.2000 \quad 0.2000)$

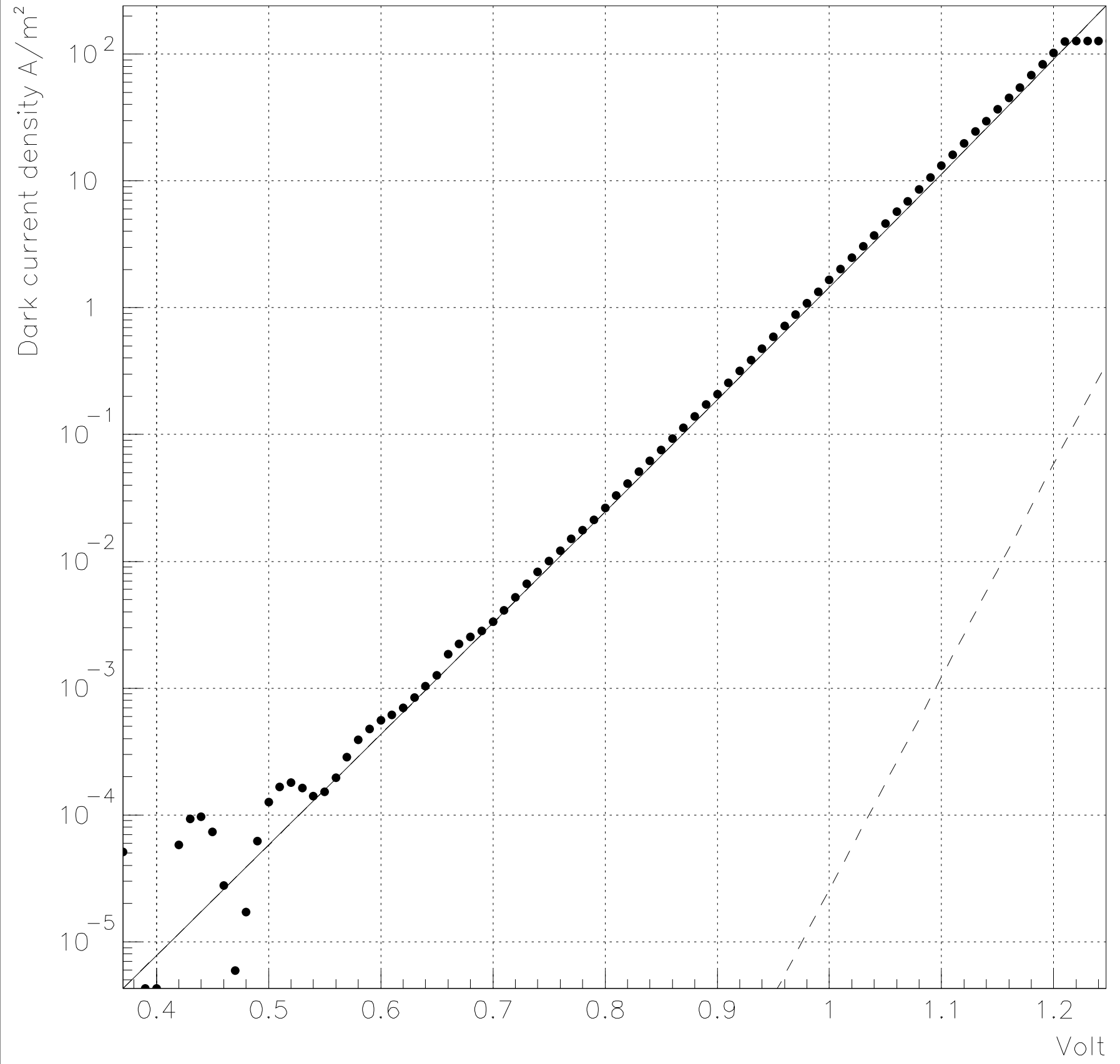


